\title{
Existence and Uniqueness Results for a Smooth Model of Periodic Infectious Diseases
}

\author{
Guy Degla ${ }^{1,2}$ \\ ${ }^{1}$ International Centre for Theoretical Physics (ICTP), Strada Costiera 11, 34151 Trieste, Italy \\ ${ }^{2}$ Institut de Mathématiques et de Sciences Physiques (IMSP), 01 BP 613 Porto-Novo, Benin
}

Correspondence should be addressed to Guy Degla; gdegla@ictp.it

Received 14 July 2016; Accepted 18 August 2016

Academic Editor: Mohsen Alimohammady

Copyright (C) 2016 Guy Degla. This is an open access article distributed under the Creative Commons Attribution License, which permits unrestricted use, distribution, and reproduction in any medium, provided the original work is properly cited.

We prove the existence of a curve (with respect to the scalar delay) of periodic positive solutions for a smooth model of CookeKaplan's integral equation by using the implicit function theorem under suitable conditions. We also show a situation in which any bounded solution with a sufficiently small delay is isolated, clearing an asymptotic stability result of Cooke and Kaplan.

\section{Dedicated to Professor Giovanni Vidossich}

\section{Introduction}

By modelling some infectious diseases with periodic contact rate that varies seasonally, Cooke and Kaplan [1] came up with the nonlinear integral equation

$$
u(t)=\int_{t-\tau}^{t} f(s, u(s)) d s, \quad-\infty<t<+\infty,
$$

where $u(t)$ represents the proportion of infections in the population at time $t, f: \mathbb{R} \times[0, \infty) \rightarrow[0, \infty) ;(t, x) \mapsto f(t, x)$ is a (nonnegative) continuous function which is $\omega$-periodic in the variable $t$; and $\tau$ is a positive real number corresponding to the length of time an individual remains infectious.

This has attracted many mathematicians such as Leggett and Williams [2], Nussbaum [3], and Agarwal and O'Regan [4] who have considered many variants of this model and used cone theoretic arguments to establish their existence results.

In this paper, we consider $\tau$ as a positive real parameter and prove under suitable conditions (5) the existence of a unique curve of periodic positive solutions when $f$ is of separable variables; say $f(t, x) \equiv q(t) g(x)$ with $q: \mathbb{R} \rightarrow$ $[0,+\infty)$ continuous and $\omega$-periodic, and $g:[0,+\infty) \rightarrow$ $[0,+\infty)$ is of class $\mathscr{C}^{1}$. Furthermore we show a uniqueness result for bounded solutions of (1) when $f(t, 0) \equiv 0, f$ is continuous and continuously differentiable with respect to its second variable $x$, and $\tau>0$ is sufficiently small.

\section{The Results}

In the sequel $\omega$ denotes a positive constant real number, $\mathscr{C}_{\omega}(\mathbb{R})$ denotes the real Banach space of $\omega$-periodic continuous functions from $\mathbb{R}$ to $\mathbb{R}$ equipped with the supremum norm

$$
\|u\|_{\omega}=\sup _{t \in \mathbb{R}}|u(t)|=\max _{0 \leq t \leq \omega}|u(t)|
$$

$\mathscr{C}_{\omega}^{1}(\mathbb{R})$ denotes the space of $\omega$-periodic continuously differentiable functions from $\mathbb{R}$ to $\mathbb{R}$, and $C_{b}(\mathbb{R})$ denotes the real Banach space of bounded continuous functions from $\mathbb{R}$ to $\mathbb{R}$ equipped with the supremum norm

$$
\|u\|_{\infty}=\sup _{t \in \mathbb{R}}|u(t)| .
$$

Given a function of two variables $u:(\tau, t) \mapsto u(\tau, t)$, we shall set

$$
u_{\tau}(t):=u(\tau, t) .
$$


Theorem 1. Let $q: \mathbb{R} \rightarrow[0,+\infty)$ be a (nonnegative) continuous $\omega$-periodic function that is not identically equal to zero and $g:[0,+\infty) \rightarrow[0,+\infty)$ be a nonnegative continuous function of class $\mathscr{C}^{1}$.

Suppose, moreover, that there exists a real number $x_{0}>0$ such that

$$
\begin{array}{r}
x_{0}-\omega \bar{q} g\left(x_{0}\right)=0, \\
\omega \bar{q}\left|g^{\prime}\left(x_{0}\right)\right|<1,
\end{array}
$$

where $\bar{q}=(1 / \omega) \int_{0}^{\omega} q(s) d s$ (the mean value of $\left.q\right)$.

Then there exists $\delta \in(0, \omega)$ and a unique curve of nontrivial nonnegative $\omega$-periodic solutions $u \in \mathscr{C}^{1}((\omega-\delta, \omega+\delta)$; $\left.\mathscr{C}_{\omega}^{1}(\mathbb{R})\right) ; \tau \mapsto u(\tau, \cdot)=: u_{\tau}$ such that by setting $u_{\tau}:=u(\tau, \cdot)$ we have

$$
u_{\omega}(t)=x_{0}, \quad \forall t \in \mathbb{R},
$$

and for each $\tau \in(\omega-\delta, \omega+\delta)$,

$$
u_{\tau}(t)=\int_{t-\tau}^{t} q(s) g\left(u_{\tau}(s)\right) d s, \quad-\infty<t<+\infty ;
$$

that is, $u_{\tau}$ solves (1) with $f(t, x) \equiv q(t) g(x)$.

Remarks 2. (i) For $\tau$ sufficiently closed but not equal to $\omega$, the solution $u_{\tau}$ provided by Theorem 1 is not constant (since it can be seen in the proof that $\left.(\partial u / \partial \tau)\left(\omega, x_{0}\right) \not \equiv 0\right)$.

(ii) The assumptions of this theorem are satisfied (due to the intermediate value theorem) when $q: \mathbb{R} \rightarrow[0,+\infty)$ is a nonnegative continuous $\omega$-periodic function that is not identically equal to zero and $g:[0,+\infty) \rightarrow[0,+\infty)$ is a nonnegative continuous function of class $\mathscr{C}^{1}$ such that

$$
\begin{aligned}
\limsup _{x \rightarrow 0^{+}} \frac{g(x)}{x} & =+\infty, \\
\inf _{x \geq x^{*}} \frac{g(x)}{x} & =0 \quad \text { for some } x^{*}>0, \\
\omega \bar{q}\left(\sup _{x>0}\left|g^{\prime}(x)\right|\right) & <1 .
\end{aligned}
$$

(iii) The conclusion of Theorem 1 still holds, according to its proof, when $q: \mathbb{R} \rightarrow[0,+\infty)$ is a nonnegative continuous $\omega$-periodic function that is not identically equal to zero, for some real number $x_{1}>0, g$ is continuously differentiable from $\left[0, x_{1}\right]$ into $[0,+\infty)$, and there exists a real number $x_{0} \in$ $\left(0, x_{1}\right)$ that satisfies the conditions (5).

(iv) Note that if $q: \mathbb{R} \rightarrow[0,+\infty)$ is a nonnegative continuous $\omega$-periodic function that is not identically equal to zero and $g:[0,+\infty) \rightarrow[0,+\infty)$ is a nonnegative continuous function of class $\mathscr{C}^{1}$ which is superlinear or for which there exists a positive number $x^{*}$ such that

$$
\begin{gathered}
g_{r}^{\prime}(0)=0, \\
\frac{g\left(x^{*}\right)}{x^{*}}>\frac{1}{\omega \bar{q}},
\end{gathered}
$$

then (1) with $\tau=\omega$ has a positive constant solution but we cannot say more because $\omega \bar{q}\left(\sup _{0<x<x^{*}}\left|g^{\prime}(x)\right|\right)>1$.
Proposition 3. Let

$$
\begin{aligned}
f: \mathbb{R} \times[0,+\infty) & \longrightarrow[0,+\infty), \\
(t, x) & \longmapsto f(t, x)
\end{aligned}
$$

be a nonnegative bounded continuous function, w-periodic with respect to $t$, not identically equal to zero and having a continuous partial derivative $\partial f / \partial x$. Suppose, moreover, that

$$
f(t, 0)=0 \quad \forall t \in \mathbb{R} .
$$

Then,

(i) for every $\tau>0$, any solution of (1) is a priori bounded,

(ii) given $\tau>0$, any solution $u$ of (1), such that

$$
\sup _{t \in \mathbb{R}} \int_{t-\tau}^{t}\left|\frac{\partial f}{\partial x}(s, u(s))\right| d s<1 \text {, }
$$

is isolated,

(iii) in particular, for any $\tau>0$ such that

$$
\sup _{t \in \mathbb{R}} \int_{t-\tau}^{t}\left|\frac{\partial f}{\partial x}(s, 0)\right| d s=\max _{0 \leq t \leq \omega} \int_{t-\tau}^{t}\left|\frac{\partial f}{\partial x}(s, 0)\right| d s<1
$$

the zero function is an isolated solution of (1).

Example 4. The assumptions of this theorem are satisfied in each of the next two cases followed by an illustration of part (iii) of Remarks 2:

(i) Let $g(x)=e^{-x}$ for every $x \geq 0$ and $q(t)=(1 / 2)(1+$ $\sin (2 \pi t))$ for all $t \in \mathbb{R}$ and $\omega=1$.

Clearly $q$ is a 1 -periodic nonnegative function with $\bar{q}=(1 / \omega) \int_{0}^{\omega} q(s) d s=1 / 2$. Moreover $g$ is a nonnegative function of class $\mathscr{C}^{1}$ on $[0,+\infty)$ and so

$$
\omega \bar{q} \sup _{x>0}\left|g^{\prime}(x)\right|=\omega \bar{q} \sup _{x>0} e^{-x}=\frac{1}{2}<1 \text {. }
$$

One can even realize that the positive solution $x_{0}$ of the equation

$$
x-\frac{e^{-x}}{2}=0
$$

belongs to the interval $(0,1 / 2)$.

(ii) Let $g(x)=\exp \left(-x^{2} / 2\right)$ for every $x \geq 0$ and $q(t)=$ $(1+\sin (\pi t)) / 2$ for all $t \in \mathbb{R}$ and $\omega=2$.

Clearly $q$ is a 2-periodic nonnegative function with

$$
\bar{q}=\frac{1}{\omega} \int_{0}^{\omega} q(s) d s=\frac{1}{2} .
$$


Moreover $g$ is a nonnegative function of class $\mathscr{C}^{1}$ on $[0,+\infty)$ and $g^{\prime}(x)=-x \exp \left(-x^{2} / 2\right)$ for $x>0$,

$$
\begin{aligned}
\lim _{x \rightarrow 0^{+}} \frac{g(x)}{x} & =+\infty \\
\lim _{x \rightarrow+\infty} \frac{g(x)}{x} & =0 \\
\omega \bar{q}\left(\sup _{x>0}\left|g^{\prime}(x)\right|\right) & =\sup _{x>0}\left(x e^{-x^{2} / 2}\right)=\left(\frac{2}{e}\right)^{1 / 2}<1 .
\end{aligned}
$$

Then we can conclude according to part (ii) of Remarks 2.

(iii) Let $g(x)=x(1-x)$ for every $0 \leq x \leq 1, q(t)=$ $5(1+\sin (4 \pi t))$ for all $t \in \mathbb{R}$, and $\omega=1 / 2$.

It follows that $q$ is a $1 / 2$-periodic nonnegative function with $\bar{q}=5$, and $g$ is a nonnegative function of class $\mathscr{C}^{1}$ on $[0,1]$ with $g^{\prime}(x)=1-2 x$ for $0<x<1$. Moreover $x_{0}=3 / 5$ satisfies

$$
\begin{aligned}
x_{0}-\omega \bar{q} g\left(x_{0}\right) & =0, \\
\omega \bar{q}\left|g^{\prime}\left(x_{0}\right)\right| & =\frac{1}{2}<1 .
\end{aligned}
$$

The result follows from part (iii) of Remarks 2.

Proof of Theorem 1. Suppose that the assumptions of Theorem 1 are satisfied.

Step 1. Let $\widetilde{g}$ be a real-valued $\mathscr{C}^{1}$-extension of $g$ to $\mathbb{R}$; for instance,

$$
\tilde{g}(x)= \begin{cases}g(x) & \text { if } x \geq 0 \\ g_{r}^{\prime}(0) x+g(0) & \text { if } x<0\end{cases}
$$

which may change sign; in other words $\widetilde{g}$ is defined from $\mathbb{R}$ into $\mathbb{R}$.

Although

$$
g([0,+\infty)) \subset[0,+\infty),
$$

we shall need just a positive real number $x_{1}>x_{0}$ such that

$$
g\left(\left[0, x_{1}\right]\right) \subset[0,+\infty)
$$

for the sake of generality (see Remarks 2(iii)). Hence

$$
\widetilde{g}(x)=g(x) \geq 0, \quad \forall x \in\left[0, x_{1}\right] .
$$

Now set

$$
\Omega=\left\{u \in \mathscr{C}_{\omega}(\mathbb{R}): 0<u(t)<x_{1}, \forall t \in[0, \omega]\right\} .
$$

Clearly $\Omega$ is open in $\mathscr{C}_{\omega}(\mathbb{R})$ and contains the constant function $x_{0}$. Moreover consider the mapping

$$
\begin{aligned}
F:(0,+\infty) \times \Omega & \longrightarrow \mathscr{C}_{\omega}(\mathbb{R}), \\
(\tau, u) & \longmapsto F(\tau, u)
\end{aligned}
$$

defined by

$$
\begin{aligned}
{[F(\tau, u)](t)=u(t)-\int_{t-\tau}^{t} q(s) \tilde{g}(u(s)) d s, } & \\
& -\infty<t<+\infty .
\end{aligned}
$$

Then $F$ is well-defined by the $\omega$-periodicity of $q$ and the continuity of both $q$ and $g$. Also for every $(\tau, u) \in(0,+\infty) \times \Omega$ fixed, we have

$$
F(\tau, u)=0 \Longleftrightarrow
$$

$$
\begin{cases}{[F(\tau, u)](t)=0,} & \forall t \in \mathbb{R} \\ u(t)-\int_{t-\tau}^{t} q(s) \tilde{g}(u(s)) d s=0, & \forall t \in \mathbb{R} \\ u(t)=\int_{t-\tau}^{t} q(s) \tilde{g}(u(s)) d s \geq 0, & \forall t \in \mathbb{R} \\ u(t)=\int_{t-\tau}^{t} q(s) g(u(s)) d s \geq 0, & \forall t \in \mathbb{R} .\end{cases}
$$

Thus for $(\tau, u) \in(0,+\infty) \times \Omega, F(\tau, u)=0$ if and only if $u$ is a positive solution of (1) with $f(t, x) \equiv q(t) g(x)$.

Step 2. Now one can see that $F$ is of class $\mathscr{C}^{1}$ by the properties of the parameter dependent integrals and those of Nemytskii operators [5].

It is not hard to check that, for every $\tau>0$ and every $u \in \mathscr{C}_{\omega}(\mathbb{R})$, we have for all $h \in \mathscr{C}_{\omega}(\mathbb{R})$,

$$
\begin{aligned}
D_{1} F(\tau, u): t \longmapsto & -q(t-\tau) g(u(t-\tau)), \\
{\left[D_{2} F(\tau, u)\right](h): t \longmapsto } & h(t) \\
& \quad-\int_{t-\tau}^{t} q(s) g^{\prime}(u(s)) h(s) d s .
\end{aligned}
$$

In particular $D_{1} F\left(\omega, x_{0}\right)$ is the function $-g\left(x_{0}\right) q$ since $q$ is $\omega$ periodic, while $D_{2} F\left(\omega, x_{0}\right)$ is the endomorphism of $\mathscr{C}_{\omega}(\mathbb{R})$; $h \mapsto D_{2} F\left(\omega, x_{0}\right)(h)$, such that

$$
\begin{aligned}
{\left[D_{2} F\left(\omega, x_{0}\right)(h)\right](t)=} & h(t) \\
& -g^{\prime}\left(x_{0}\right) \int_{t-\omega}^{t} q(s) h(s) d s,
\end{aligned}
$$

$\forall t \in \mathbb{R}$.

Step 3. We have $F\left(\omega, x_{0}\right)=0$.

Moreover

$$
\begin{aligned}
\left\|D_{2} F\left(\omega, x_{0}\right)-I\right\| & =\sup _{\|h\|_{\omega} \leq 1}\left\|D_{2} F\left(\omega, x_{0}\right)(h)-h\right\|_{\omega} \\
& \leq \sup _{t \in \mathbb{R}}\left|g^{\prime}\left(x_{0}\right)\right| \int_{t-\omega}^{t} q(s) d s \\
& =\omega \bar{q}\left|g^{\prime}\left(x_{0}\right)\right|<1,
\end{aligned}
$$

showing that $D_{2} F\left(\omega, x_{0}\right)$ is an isomorphism of $\mathscr{C}_{\omega}(\mathbb{R}), \mathrm{Cf}[5$, page 212 ] or [6, page 31 ]. 
Therefore by the implicit function theorem [5-7], there is an open neighbourhood $V_{0}$ of $\left(\omega, x_{0}\right)$ in $(0,+\infty) \times \Omega$, a positive real number $\delta<\omega$, and an open neighbourhood $\Omega_{0} \subseteq \Omega$ and a unique continuously differentiable map $\varphi$ from $(\omega-\delta, \omega+\delta)$ to $\Omega_{0}$ such that $\varphi(\omega)=x_{0}$ and for any $(\tau, u) \in(\omega-\delta, \omega+\delta) \times \Omega$,

$$
\begin{gathered}
\left((\tau, u) \in V_{0}, F(\tau, u)=0\right) \Longleftrightarrow \\
(\tau \in(\omega-\delta, \omega+\delta), u=\varphi(\tau)) .
\end{gathered}
$$

In addition

$$
\varphi^{\prime}(\tau)=\left[D_{2} F(\tau, \varphi(\tau))\right]^{-1} D_{1} F(\tau, \varphi(\tau)), \quad \forall \tau \in U_{0},
$$

and so

$$
\varphi^{\prime}(\omega)=-g\left(x_{0}\right)\left[D_{2} F\left(\omega, x_{0}\right)\right]^{-1}(q) \not \equiv 0 .
$$

The result follows.

Proof of Proposition 3. (1) Let us fix $\tau>0$ and suppose that $v$ is any solution of (1) with $f$ satisfying the hypotheses of Proposition 3. Then we have

$$
0 \leq v(t)=\int_{t-\tau}^{t} f(s, v(s)) d s \leq \tau\|f\|_{\infty}, \quad \forall t \in \mathbb{R},
$$

showing that $v$ is bounded by the boundedness of $f$.

(2) Let us fix $\tau>0$ and suppose $u$ is a solution of (1) such that

$$
\sup _{t \in \mathbb{R}} \int_{t-\tau}^{t}\left|\frac{\partial f}{\partial x}(s, u(s))\right| d s<1
$$

Consider the nonlinear map $G: C_{b}(\mathbb{R}) \rightarrow C_{b}(\mathbb{R})$ defined by

$$
[G(v)](t)=v(t)-\int_{t-\tau}^{t} f(s, v(s)) d s, \quad \forall t \in \mathbb{R} .
$$

Indeed if $v$ is a bounded continuous function from $\mathbb{R}$ into $\mathbb{R}$, then $G(v)$ is also continuous by the continuity of $f$ and is moreover bounded by the previous result.

Again it is not hard to see that $G$, as a map from $C_{b}(\mathbb{R})$ into $C_{b}(\mathbb{R})$, is continuously differentiable and given $v \in C_{b}(\mathbb{R})$, we have for every $h \in C_{b}(\mathbb{R})$

$$
\left[G^{\prime}(v)(h)\right](t)=h(t)-\int_{t-\tau}^{t} \frac{\partial f}{\partial x}(s, v(s)) h(s) d s,
$$

$\forall t \in \mathbb{R}$.

So that

$$
\left\|G^{\prime}(u)-I\right\| \leq \sup _{t \in \mathbb{R}} \int_{t-\tau}^{t}\left|\frac{\partial f}{\partial x}(s, u(s))\right| d s<1
$$

by assumption. This implies that $G^{\prime}(u)$ is an automorphism. And since $G(u)=0$, we conclude that $u$ is an isolated zero of $G$; that is, $u$ is an isolated solution of (1).

(3) follows immediately from (2).

\section{Competing Interests}

The author declares that there are no competing interests regarding the publication of this paper.

\section{Acknowledgments}

The author is very grateful to the Abdus Salam International Centre for Theoretical Physics (ICTP, Trieste, Italy) for its hospitality and financial support during his 2016 visit as a Regular Associate.

\section{References}

[1] K. L. Cooke and J. L. Kaplan, "A periodicity threshold theorem for epidemics and population growth," Mathematical Biosciences, vol. 31, no. 1-2, pp. 87-104, 1976.

[2] R. W. Leggett and L. R. Williams, "A fixed point theorem with application to an infectious disease model," Journal of Mathematical Analysis and Applications, vol. 76, no. 1, pp. 91-97, 1980.

[3] R. Nussbaum, "A periodicity threshold theorem for some nonlinear integral equations," SIAM Journal on Mathematical Analysis, vol. 9, no. 2, pp. 356-376, 1978.

[4] R. P. Agarwal and D. O'Regan, "Periodic solutions to nonlinear integral equations on the infinite interval modelling infectious disease," Nonlinear Analysis: Theory, Methods \& Applications, vol. 40, no. 1-8, pp. 21-35, 2000.

[5] H. Amann and J. Escher, Analysis II, Birkhäuser, 1999.

[6] A. Ambrosetti and G. Prodi, A Primer of Nonlinear Analysis, vol. 34 of Cambridge Studies in Advanced Mathematics, Cambridge University Press, 1993.

[7] A. Ambrosetti and A. Malchiodi, Nonlinear Analysis and Semilinear Elliptic Problems, vol. 104 of Cambridge Studies in Advanced Mathematics, Cambridge University Press, 2007. 


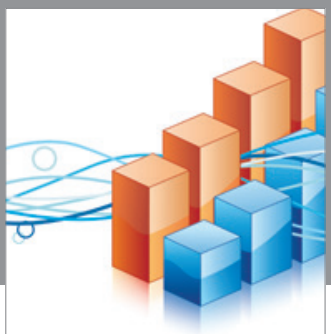

Advances in

Operations Research

vatem alat4

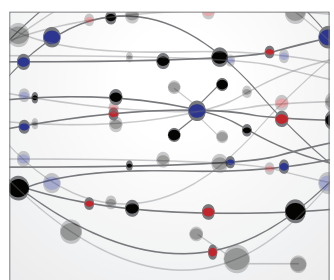

\section{The Scientific} World Journal
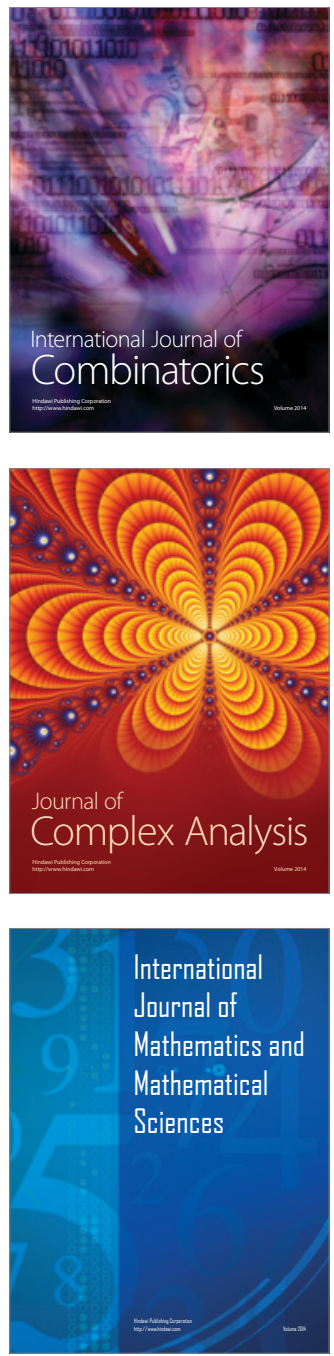
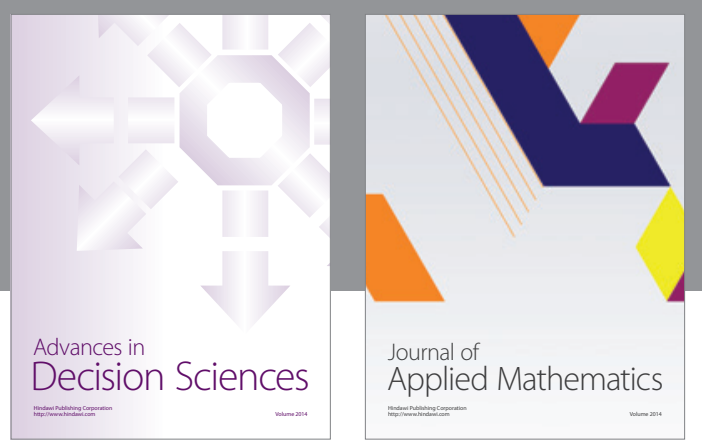

Algebra

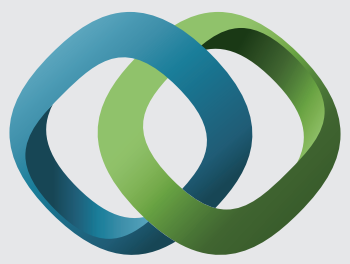

\section{Hindawi}

Submit your manuscripts at

http://www.hindawi.com
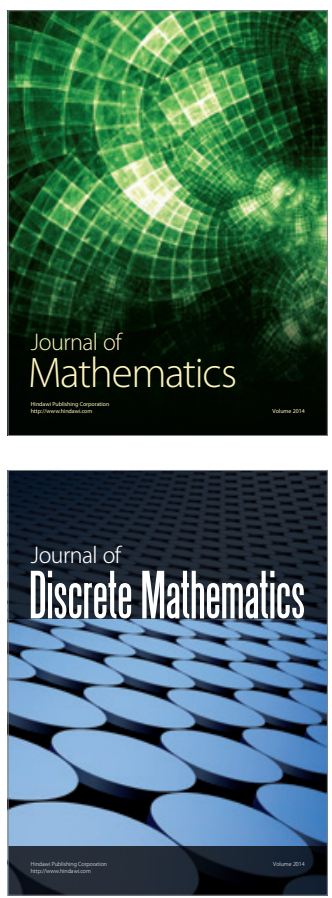

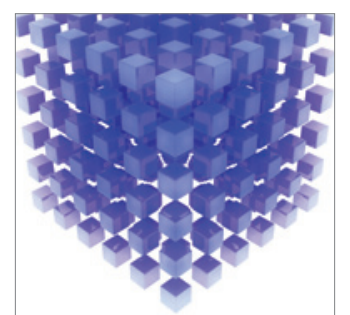

Mathematical Problems in Engineering
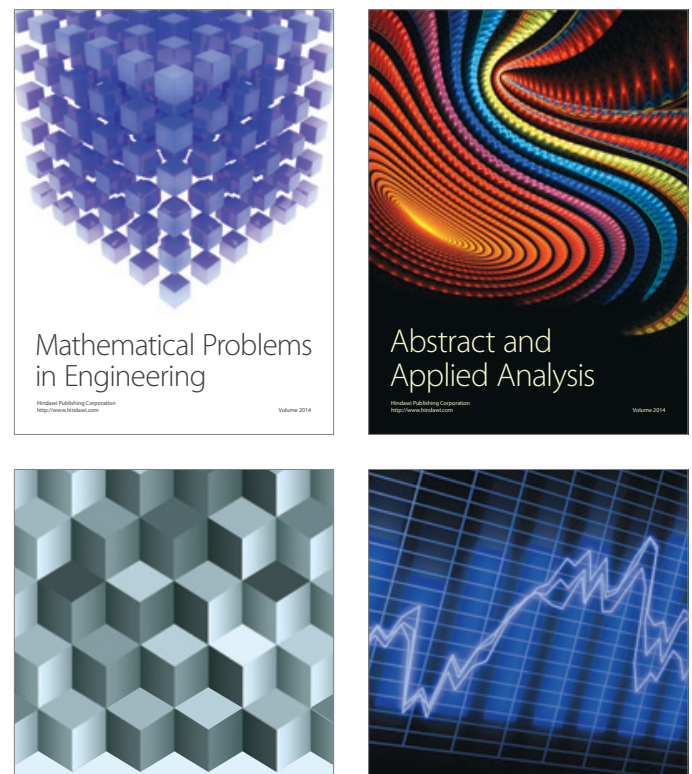

Journal of

Function Spaces

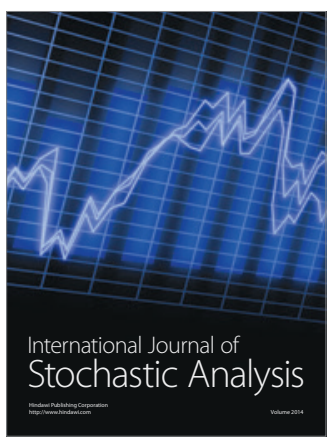

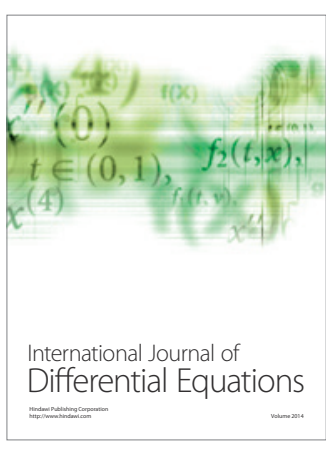
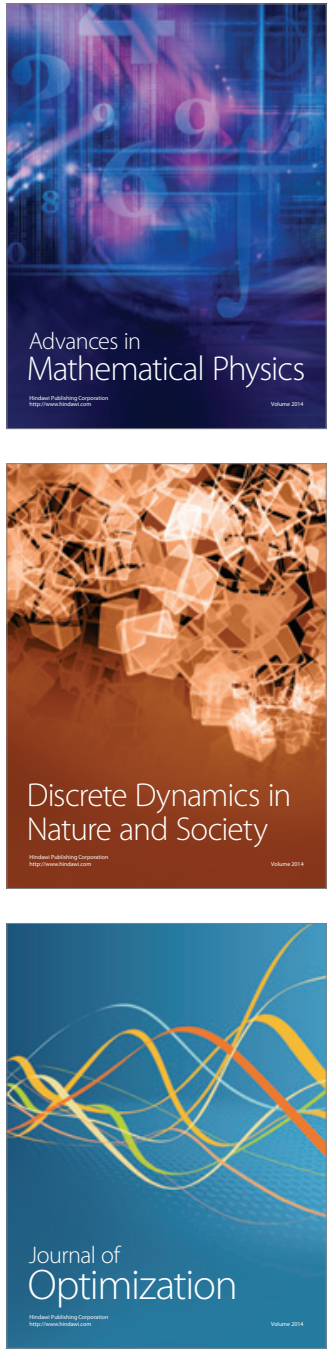